\title{
La genèse contrariée de Belle du Seigneur : le projet déjoué d'une "Geste des Juifs »
}

\section{Maxime Decout}

\section{(2) OpenEdition}

\section{Journals}

Édition électronique

URL : http://journals.openedition.org/genesis/375

DOI : 10.4000/genesis.375

ISSN : 2268-1590

\section{Éditeur :}

Presses universitaires de Paris Sorbonne (PUPS), Société internationale de génétique artistique littéraire et scientifique (SIGALES)

\section{Édition imprimée}

Date de publication : 10 octobre 2010

Pagination : 141-150

ISBN : 978-2-84050-711-6

ISSN : 1167-5101

\section{Référence électronique}

Maxime Decout, "La genèse contrariée de Belle du Seigneur : le projet déjoué d'une « Geste des Juifs » », Genesis [En ligne], 31 | 2010, mis en ligne le 24 septembre 2012, consulté le 01 mai 2019. URL: http://journals.openedition.org/genesis/375; DOI : 10.4000/genesis.375 


\title{
La genèse contrariée de Belle du Seigneur : le projet déjoué d'une «Geste des Juifs »
}

\author{
Maxime Decout
}

$\mathrm{T}$ Ooute ÉTUde GÉNÉTIQue SUR COHEN se heurte à une difficulté majeure : l'écrivain, déçu à la lecture de la correspondance de Proust après sa mort, a voulu soigneusement contrôler l'image de soi qu'il léguerait à la postérité, apposant le sceau du secret sur tout ce qui pouvait le menacer, les coulisses de sa vie comme celles de son œuvre. En effet la statue de l'idole qu'était Proust fut, d'un coup, renversée, révélant un homme mondain et flagorneur, peu différent somme toute d'un Adrien Deume, fat et inconsistant. Aussi, conformément à ses volontés testamentaires, son épouse, Bella Cohen, refusant de jouer aux «testaments trahis ", a pris soin de détruire l'ensemble des brouillons des romans. L'atelier de travail de Cohen a alors été condamné au vide-ordures par contumace testamentaire. Dès lors, la question de la genèse de l'œuvre pourrait passer pour terra incognita et être définitivement considérée comme nulle et non avenue. Pourtant, il existe un certain nombre de témoignages comme d'indices laissés au cours de la rédaction qui peuvent servir de guides pour retracer le cheminement d'une pensée et d'une écriture.

La singularité de la conception du roman chez Cohen réside d'abord dans l'unité, autant thématique que stylistique, qui lie les quatre romans, Solal, Mangeclous, Belle du Seigneur et Les Valeureux, entre eux ${ }^{1}$. Le romancier avait en effet pour projet d'écrire une vaste " geste des Juifs 2 » à travers les aventures de Solal. Pourtant, plus qu'un cycle à proprement parler, une « geste » épique, les textes, si ce n'est le premier roman, Solal, paru en 1930, semblent avoir été pensés comme une entité dont le morcellement en romans indépendants ne résulte que de césures éditoriales partiellement indépendantes de la volonté du créateur. C'est pourquoi Cohen avait coutume de se déclarer l'auteur d'une seule œuvre. Un seul livre, inlassablement recommencé, réentrepris de 1930 à 1968 : Belle du
Seigneur. La curiosité génétique n'a dès lors ici rien d'obscène, car, même si elle fait entorse au souhait de l'écrivain, elle s'inscrit dans une quête herméneutique qui permettra de constater que le grand roman de Cohen n'a jamais été conçu comme tel par l'auteur. Car ce n'est qu'après une genèse contrariée par de nombreuses péripéties éditoriales que Belle du Seigneur nous est parvenu en l'état, biaisant définitivement les lectures qui en ont été faites, notamment par une atténuation manifeste du comique que Cohen avait prévu d'y inclure. Dès lors, il s'agira de mieux saisir les répercussions des coupes multiples qui ont pu être réalisées dans l'œuvre afin d'éclairer la structure singulière des différents textes, tout particulièrement de Mangeclous ou des Valeureux dont l'autonomie en tant que romans seuls fait problème. La structure de ces textes, précisée à la lumière de la genèse contrariée du livre unique qu'était au départ Belle du Seigneur, apporte en effet un éclairage nécessaire sur leur signification.

\section{Mangeclous : une création par retranchement}

Le destin de l'œuvre romanesque de Cohen a en effet été très singulier. Après la publication de Solal en 1930, se dessine une vaste entreprise qui ne pourra pas prendre forme réellement, celle d'écrire un livre Un, une " geste des Juifs ». Mangeclous (1938), Belle du Seigneur (1968) et Les Valeureux (1969) sont en fait un texte unique qui n'a été morcelé qu'à cause des aléas de la publication.

1. Solal, Paris, Gallimard, coll. « Folio », 1981 [1930, renouvelé en 1958] ; Mangeclous (M), Paris, Gallimard, coll. « Folio », 1980 [1938, renouvelé en 1969] ; Belle du Seigneur (BS), Paris, Gallimard, coll. «Folio », 1998 [1968] ; Les Valeureux (V), Paris, Gallimard, coll. « Folio », 1986 [1969].

2. Jean Blot, Albert Cohen, Paris, Balland, 1986, p. 249. 
Dès les années trente, le projet s'ébauche dans l'esprit de l'écrivain. À cette époque, a déjà eu lieu la grande rencontre de sa vie avec l'œuvre de Marcel Proust. Comme le modèle vénéré, Cohen rêve d'une œuvre totale, tentaculaire, mais en un seul vaste volume. Il conçoit très rapidement la structure globale de son texte. La charpente de l'œuvre préexiste ainsi aux publications des divers volumes à la manière du roman de Proust dont l'architecture d'ensemble était déjà prévue avant la publication des différents tomes 3 .

Après la publication de «Projections ou Après-minuit à Genève ${ }^{4}$ » en 1922 à la N.R.F., Jacques Rivière, alors directeur de la revue, prend contact avec Cohen pour lui offrir un contrat pour cinq romans à paraître. Gallimard lui alloue alors une rente 5 . L'écrivain n'a qu'à s'engager pour un premier texte qu'il décide d'appeler « Rapides internationaux », dans la mouvance de Paul Morand qui l'impressionnait alors. Ce titre est bien loin de ce que l'auteur écrira par la suite mais est révélateur quant aux premières préoccupations de Cohen dans la droite ligne de ce qu'il vient de publier dans la N.R.F6. Huit ans après, Solal est enfin publié. Le projet d'ensemble commence à voir le jour dans l'esprit de Cohen. Pourtant, huit ans après Solal, Cohen n'a toujours pas présenté le roman suivant attendu par Gallimard, qui s'impatiente. L'écrivain est contraint de s'éloigner quelque peu de son projet initial. Et pourtant, son manuscrit se monte déjà à quelque trois mille pages 7 . Mais rien n'est prêt. Dans la hâte, il soustrait de nombreux passages concernant les Valeureux pour les regrouper dans un livre à dominante comique où Solal et Ariane ne tiendront que peu de place. Anne-Marie Boissonnas, qui a été sa secrétaire et a tenu un journal pendant la rédaction des romans, précise que ces textes sont extraits du premier livre de Belle du Seigneur qui allait jusqu'au départ des amants pour Agay $^{8}$. Si l'on devait restaurer l'intégralité du projet de départ à partir des textes publiés, l'ensemble des épisodes de Mangeclous se situerait donc avant qu'Ariane et Solal ne partent pour Agay, c'est-à-dire lorsque l'amour en est encore à ses débuts enchanteurs. L'écrivain souhaitait vraisemblablement publier très rapidement la suite qui était alors annoncée sur la page titre de l'édition originale de Mangeclous, même si cette solution de demi-mesure ne pouvait le satisfaire pleinement. Mais la guerre qui approche en empêche Cohen, lui qui doit alors fuir pour l'Angleterre en abandonnant à Paris l'ensemble de son manuscrit. Retracer ce cheminement nous amène alors à nous interroger, comme le dit Louis Hay, sur « ce qu'une œuvre aurait pu être et ce qu'elle est » afin d'éclairer le texte « à la lumière des possibles qu'il a expérimentés 9 ».

Autour de la préparation de Mangeclous à partir du premier manuscrit, c'est alors un véritable chantier qui s'organise par équipe. Anne-Marie Boissonnas et Marianne, deuxième épouse de l'écrivain, corrigent les épreuves. Il faut taper sans arrêt de nouvelles pages, les corriger, les taper à nouveau. Sa fille, Myriam Cohen, aide l'écrivain en l'écoutant et c'est en fait elle qui tient le rôle du destinataire féminin, toujours présent lorsque Cohen écrivait, dictant tous ses textes à une femme. Ensemble, le rire est au rendez-vous. Ce destinataire privilégié, mais pas unique, puisque Cohen travaille avec sa fille sur de nombreux passages déjà écrits dans le vaste manuscrit préparatoire, a certainement eu une influence considérable sur la tonalité du texte10. Celui-ci est en effet, beaucoup plus que tous les autres et même beaucoup plus que Les Valeureux d'apparence lui aussi relativement comique, dominé par la légèreté et l'humour.

3. Voir par exemple Gérard Genette, Seuils, Paris, Le Seuil, coll. « Points essais », 1987, p. 66-68, qui retrace succinctement les aléas de la publication chez Proust et le choix difficile du titre d'ensemble de l'œuvre afin de la rassembler par-delà la fragmentation éditoriale.

4. Albert Cohen, «Projections ou Après-minuit à Genève », N.R.F., 10e année, 11e série, ${ }^{\circ} 109$, 1er octobre 1922, p. 414-446.

5. Gérard Valbert, Albert Cohen, le Seigneur, Paris, Grasset, 1990, p. 302.

6. « Projections ou Après-minuit à Genève » est un texte influencé par la vie du jeune écrivain à Genève. Le cosmopolitisme est omniprésent, les milieux de la politique internationale sont extrêmement représentés.

7. Gérard Valbert, Albert Cohen, Le Seigneur, op. cit., p. 203-303.

8. Anne-Marie Boissonnas-Tillier, «À propos de la première version de Belle du Seigneur (1935-1938) », Cahiers Albert Cohen, $\mathrm{n}^{\circ} 2,1992$, p. 20.

9. Louis Hay, La Littérature des écrivains. Questions de critique génétique, Paris, José Corti, coll. « Les essais », 2002, p. 29.

10. Cohen a plusieurs fois affirmé avoir écrit Mangeclous pour faire rire sa fille. La réalité est plus complexe. Dans un de ses entretiens il dit d'ailleurs : "Mangeclous pour une autre merveilleuse et aussi un peu pour faire rire ma fille» («Albert Cohen : "Tous mes livres ont été écrits par amour" », entretien avec Jean-Jacques Brochier et Gérard Valbert, Le Magazine littéraire, $\mathrm{n}^{\circ}$ 174, avril 1979). La «merveilleuse » dont il s'agit est de toute évidence Marianne. 
Par ailleurs, la précipitation dans laquelle ont été assemblés, écrits ou réécrits les chapitres de Mangeclous a informé la facture toute particulière de l'œuvre. L'intrigue est suivie mais elle n'en est pas moins globalement constituée par de petits épisodes, très lâchement reliés les uns aux autres, et qui présentent une certaine autonomie. Les deux tiers du roman mettent uniquement en scène les Valeureux. Et Cohen a délibérément décidé d'inclure à la fin quelques chapitres introducteurs des aventures d'Ariane et de Solal. Solal n'apparaît dans le texte que tardivement $(M, 223)$ et la première mention d'Ariane se fait attendre (282). Lorsque le héros part enfin épier les Deume chez eux (331), c'est là que commence véritablement Belle du Seigneur. C'est pourquoi la fin du roman signe l'éviction des Valeureux : Ariane et Solal deviennent les personnages principaux. La lecture de Mangeclous seul a donc de quoi surprendre pour qui ne connaîtrait pas l'existence de Belle du Seigneur. Amputée de sa suite, l'œuvre perd de sa cohérence. Tout est finalement laissé en suspens. L'intrigue n'a pas de fin, le voyage des Valeureux est inachevé. Le roman se termine sur une histoire qui vient se greffer à lui de façon incompréhensible, comme une excroissance exogène sans rapport avec l'organisme sur lequel elle s'insère. Nul dénouement. L'excipit est en fait un incipit. Voilà de quoi déconcerter le lecteur. La lecture de Mangeclous nécessite donc de le replacer dans son contexte. Son propos s'éclaire lorsque l'on peut restaurer sa continuité avec Belle du Seigneur.

Cohen commente même dans son texte cette structure, comme pour prévenir les réactions du lecteur. Il présente Mangeclous comme un intermède dans l'histoire de Solal et d'une femme où, progressivement, les Valeureux ont pris toute la place. Dans un passage de l'édition de 1938, retranché par la suite, on peut lire cette justification de l'auteur :

Comme on a pu le voir, ce roman est un intermède dont Solal est le plus souvent absent. Je me suis résigné à laisser gambader mes Valeureux en toute liberté pendant trente jours. En commençant j'avais l'intention de leur mesurer la place et de ne leur concéder que trois ou quatre chapitres - le reste du livre devant être consacré à Solal et à une femme. Mais ils se sont fourrés partout. Et ces valets sont devenus les maîtres du jeu 11 .
La fragmentation du texte s'explique ainsi par la prolifération des compères céphaloniens. Et, dans le souci de rendre visibles les liens qui doivent structurer la « geste des Juifs » toujours en projet, l'existence de Solal est plusieurs fois rappelée pour y renvoyer le lecteur $(M, 46,49)$. Les contraintes éditoriales et le temps qui manque sont, de la même manière, mis en lumière comme pour se dédouaner par avance, expliquant pour part la multiplication des métalepses explicatives qui rompent régulièrement l'illusion romanesque :

Qu'on excuse la manière peu ordonnée dont je vais parler. Mais ce chapitre est écrit au dernier moment et le manuscrit doit être remis demain à l'éditeur (48).

Pour prévenir le lecteur, la jaquette du livre annonce d'ailleurs la future publication de Belle du Seigneur. L'édition anglaise avait de son côté bien compris l'étrangeté d'un tel texte. C'est pourquoi elle avait supprimé toute la fin, c'est-à-dire les chapitres relatifs à Ariane et Solal afin d'assurer une cohérence plus globale au livre12.

\section{Belle du Seigneur amputé une deuxième fois}

Mais ce n'est que trente ans après Mangeclous que le lecteur a enfin pu lire la suite des aventures d'Ariane et Solal, en 1968. On comprend bien dans ce contexte que les quelques lecteurs se souvenant du roman de 1938 se comptaient alors sur les doigts d'une main. Belle du Seigneur ne pouvait qu'être lu de façon autonome, sans prendre en considération les deux textes qui l'avaient précédé et qui pourtant ont un rôle fondamental pour sa compréhension. La perception d'un ensemble romanesque cohérent s'en trouvait évidemment empêchée. Nous devons dès lors prendre au sérieux ce qu'Antoine Compagnon appelle « l'influence retardée, les contretemps du goût, l'imprévisibilité de la réception,

11. Albert Cohen, Euvres, édition établie par Christel Peyrefitte et Bella Cohen, Paris, Gallimard, coll. "Bibliothèque de la Pléiade », 1993, p. 1279. Ce volume contient: Paroles juives, Solal, Mangeclous, Le Livre de ma mère, Ézéchiel, Les Valeureux, Ô vous frères humains, Carnets 1978 et « Churchill d'Angleterre ».

12. Albert Cohen, Mangeclous, London, Routledge, 1940. 
et la reconnaissance $\operatorname{tardive}^{13} \gg$. Car le ton incisif et caustique de Mangeclous, ses diatribes acides sur la passion amoureuse n'étaient dans l'esprit de personne : la dénonciation de l'amour, au cœur de Belle du Seigneur, s'en trouvait nécessairement affaiblie, faute de pouvoir établir les parallèles nécessaires entre deux textes complémentaires. Cela a donc favorisé les lectures sans demi-mesure qui ont été faites de Belle de Seigneur comme roman d'amour. Il est par exemple raisonnable de supposer que la virulente critique des amours d'Anna Karénine et de Wronski faite par Mangeclous (M, 137 sq.) devait initialement prendre place avant la scène de séduction au Ritz au cours de laquelle Solal stigmatise lui aussi les séducteurs et leurs méthodes (BS, 405-439).

Le déroulement précis de la publication du texte est d'ailleurs assez significatif quant à la structure finale du roman. En effet, trente ans après la publication de Mangeclous, Cohen n'a toujours pas présenté le fameux roman auquel il travaille depuis si longtemps et que Gallimard attend encore. Dans l'intervalle, l'auteur a trouvé un regain de célébrité avec la publication du Livre de ma mère en 1954. Mais la pression de l'éditeur monte. Cohen se voit derechef dans la nécessité de travailler de façon précipitée. Il achève très rapidement son Belle $d u$ Seigneur qu'il présente à Gallimard. Le manuscrit contient alors à peu près mille cinq cents pages. L'éditeur prend peur à la vue de cet imposant volume. C'est inéditable. Le livre a des proportions énormes et ne pourra que décourager le public. Pourtant Cohen refuse de scinder son texte en deux volumes, il souhaite que l'histoire d'Ariane et Solal soit un tout. De ce fait, il doit modifier l'architecture de l'œuvre.

Il retire donc encore une fois de son manuscrit plusieurs passages concernant les Valeureux. Le scénario qu'il a connu en 1938 se réitère. Le nouveau texte présenté à Gallimard reçoit son approbation. Belle du Seigneur est publié en 1968 et après son succès considérable, les passages retranchés sont publiés l'année suivante dans Les Valeureux. Comme pour Mangeclous, l'auteur a besoin d'intervenir dans le texte pour commenter la forme du livre. Ainsi explique-t-il à son lecteur que « les événements contés dans ce livre sont antérieurs à ceux qui sont rapportés dans Belle du Seigneur» $(V, 33)$. On voit donc à quel point les contraintes éditoriales ont modifié le texte de départ. L'alternance entre les chapitres sur les Valeureux et ceux sur Ariane et Solal se voit en partie supprimée. L'équilibre de l'œuvre entre ces deux pôles est détruit. C'est d'ailleurs ce qui a amené certains commentateurs à scinder en deux massifs distincts l'œuvre de Cohen, opposant alors le volet « sérieux » constitué par les aventures amoureuses de Solal (Solal, Belle du Seigneur), et le volet « comique » fait des aventures des Valeureux (Mangeclous, Les Valeureux) ${ }^{14}$. Mais la construction étrange de Mangeclous avertissait déjà le lecteur que cette opposition n'était qu'un leurre. Comme nous l'avons mentionné, Mangeclous a été constitué grâce aux fragments retranchés de Belle du Seigneur, tous situés avant le départ pour Agay. Ces chapitres appartiennent donc aux débuts amoureux et leur tonalité s'en ressent nécessairement. En revanche, la tonalité des Valeureux est beaucoup moins alerte que celle de Mangeclous. Le climat y est plus sombre. Il est en effet possible que les contraintes éditoriales aient joué à ce niveau aussi et que la guerre ait laissé son empreinte.

Sommes-nous donc entièrement livrés aux suppositions quand nous souhaitons avoir au moins un pressentiment de ce qu'aurait pu être l'architecture du texte intégral ? Pas exactement. Car une lecture attentive de Belle du Seigneur peut nous renseigner sur la position de certains chapitres des Valeureux. Il est en effet assez surprenant de voir apparaître, au chapitre XII, ces mots dans la bouche de Mangeclous : « en ma qualité d'ancien recteur de l'Université israélite et philosophique de Céphalonie » (BS, 143). Comment comprendre de tels propos pour le lecteur de 1968 ? Assurément, personne n'aura relevé, attribuant cette saillie à la personnalité excentrique du Valeureux. Mais Cohen récidive en qualifiant à nouveau le personnage d'ancien recteur au chapitre XXIV (273).

13. Antoine Compagnon, « Histoire littéraire ou histoire des auteurs?», dans Modernités, $\mathrm{n}^{\circ}$ 18, «L'auteur entre biographie et mythobiographie », Bordeaux, Presses universitaires de Bordeaux, 2002, p. 32.

14. Hubert Nyssen suggère dans Lecture d'Albert Cohen, Avignon, Alain Barthélemy/Actes Sud, 1981, p. 26, de lire Les Valeureux avant Belle du Seigneur, «sous peine de ne pas bénéficier de la perspective que crée la dérision ». Mais il serait plus exact de dire que la lecture des Valeureux et celle de Mangeclous devrait être entrelacée au sein de celle de Belle du Seigneur pour retrouver la composition originelle, ce qui bien entendu est impossible. 
Pourtant Mangeclous ne sera recteur qu'en 1969, dans le livre suivant. Ces mots, pour incongrus qu'ils sont dans l'œuvre de 1968, sont, selon toute vraisemblance, des « coquilles » que Cohen n'a pas relevées après l'éviction des chapitres destinés aux Valeureux. Ils font certainement partie des infimes détails trahissant l'état premier du texte et qui ont échappé à la vigilance de l'auteur. Or l'épisode de l'université se déroule des chapitres IX à XII $(V$, 106175) dans le livre de 1969. Il est donc raisonnable de supposer que tous ces chapitres devaient se tenir avant le chapitre XII de Belle du Seigneur, donc relativement au début du texte, mais surtout avant la scène de séduction au Ritz (BS, $405 s q$.). Ainsi les différents points exposés par Solal dans la scène de séduction, appelés les « manèges » du séducteur, prennent un éclat bien plus ironique à la lumière des « manœuvres » consciencieusement présentées par le Céphalonien dans son université ( $V$, chap. XI), manœuvres qui auraient dû être décrites au moins avant le chapitre XII de Belle du Seigneur, c'est-à-dire quelque deux cent cinquante pages avant la scène de séduction au Ritz. Les deux textes se répondent point par point, avec un parallélisme assez strict entre les manèges exposés par Solal et les manœuvres de Mangeclous ${ }^{15}$.

De plus, dans Belle du Seigneur, nous ne voyons pas arriver les Valeureux depuis leur île : ils apparaissent immédiatement à Genève pour venir voir Solal. Or, après l'épisode de l'université dans Les Valeureux se déroule le long voyage des cousins jusqu'en Europe pour rejoindre le héros. Ils passent par Rome, Paris, et Mangeclous fausse compagnie aux autres pour se rendre à Londres. Il semble donc probable que toute la partie se déroulant à Céphalonie ainsi que le voyage vers l'Europe devait se tenir au début de Belle du Seigneur, structure qui était certainement aussi celle du manuscrit des années trente et que l'on retrouve dans Mangeclous, où les scènes avec les Valeureux à Céphalonie devaient se dérouler avant que l'aventure amoureuse ne commence vraiment.

\section{De Mangeclous aux Valeureux : l'origine d'un ressassement}

Par ailleurs, un dernier point mérite d'être souligné : la redondance de certains épisodes entre Mangeclous d'une part et Les Valeureux d'autre part. L'incipit est symétrique : tout commence tôt le matin à Céphalonie avec une description paradisiaque de l'île et un zoom sur un seul Valeureux : Salomon $(M, 11)$ et Mangeclous $(V, 11)^{16}$. La suite des deux romans est parfaitement parallèle : la présentation des Valeureux ( $M, 46-52$; $V$, 79-91), le cours de séduction sur Anna Karénine ( $M, 130-148 ; V, 129-175)$, les repas de Mangeclous avec ses enfants $(M, 71-80 ; V, 63-78)$, la vie dans le ghetto, les bonnes affaires de Mangeclous $(M, 83-95 ; V, 11-54)$, la lettre de Solal et le secret qui provoquent leur départ pour Genève $(M, 26-45,96-114 ; V, 176-189)$, le voyage, avec un épisode sur le bateau où les Valeureux discutent ( $M, 118$ sq. ; V, 221-268), l'imposture de Mangeclous qui par deux fois fausse compagnie à ses cousins pour les devancer $(M, 269-284 ; V, 288)^{17}$. Comment rendre compte de cette étonnante symétrie des deux textes ? Pourquoi Cohen semble-t-il, à trente années de distance, réécrire la même histoire ? C'est ainsi qu'Alain Schaffner a par exemple pu démontrer avec finesse que les deux textes se déroulaient en même temps ${ }^{18}$. Mais, plus qu'à imputer cette simultanéité à une «négligence » ou à la création d'un « hors temps », tout porte à croire que Cohen tenait vraiment à la structure symétrique des deux textes qui, dans Mangeclous, menait les Valeureux depuis la case Céphalonie jusqu'à Genève pour rencontrer Solal. Si l'on considère Les Valeureux comme devant être globalement situé au début de Belle du Seigneur, on constate alors que la structure du projet global de 1968 (Les Valeureux-Belle du Seigneur) est la même que

15. Le parallèle entre les deux textes a été finement étudié, mais sans tenir compte des données concernant la genèse du texte, par Denise Goitein-Galperin, «Deux discours de séduction : "Manèges" et "Manœuvres" ", Cahiers Albert Cohen, n 5, 1995, p. 103-124.

16. L'incipit de Solal est lui aussi bâti sur le même schéma, mais cette fois c'est Saltiel qui est seul. Les trois plus importants cousins se voient ainsi accorder à chacun un incipit

17. Dans le détail des textes, les symétries sont là encore extrêmement prégnantes. À titre d'échantillon, nous relèverons simplement cette sentence sur l'amour : «quelle valeur accorder à un sentiment si fragile qu'un léger vent suffit à l'abattre et le flétrir ? » $(M, 140)$ qui devient par la suite : «quel sérieux accorder à un sentiment que le moindre vent suffit à flétrir ? » $(V, 172)$. De même, la harangue contre les romanciers mensongers est présente dans les deux textes où reviennent les mêmes formulations $(M, 140-141 ; V, 172)$.

18. Alain Schaffner, Le Goût de l'absolu. L'enjeu sacré de la littérature dans l' euvre d'Albert Cohen, Paris, Champion, coll. " Littérature de notre siècle », 1999, p. 302. 
celle du projet de 1938 (Mangeclous-Belle du Seigneur). Le redoublement des épisodes entre Mangeclous et Les Valeureux est donc certainement dû au fait que Cohen, n'ayant pu réaliser la structure prévue en 1938, ait tenté de la réinvestir en 1968. Mais là encore, elle ne pourra se concrétiser. Il est donc essentiel d'envisager comme particulièrement signifiant ce cheminement qui déplace le lecteur depuis Céphalonie à l'Europe, par le médium des Valeureux, jusqu'à la rencontre des amants en Occident. Ainsi, cet itinéraire reconstruit indique clairement la signification de la topographie romanesque, avec ses passerelles Orient-Occident et ses territoires exclusifs.

De plus, dans ce parallélisme évident de deux projets avortés (Mangeclous-Belle du Seigneur et Les ValeureuxBelle du Seigneur) se dessinent des obsessions fortes de Cohen, accompagnées de leurs implications toujours identiques sur les choix structuraux qui président à la construction du texte. L'œuvre a été conçue dès 1938 selon une structure évidente qui faisait précéder l'aventure amoureuse du couple d'un exposé par Mangeclous des ficelles de la passion amoureuse et de ses codes, que ce soit dans Mangeclous pour la version de 1938 (Mangeclous-Belle du Seigneur) ou dans Les Valeureux pour la version de 1968 (Les Valeureux-Belle du Seigneur). La répétition du cours de Mangeclous sur la passion à travers l'exemple d'Anna Karénine dans Mangeclous et dans Les Valeureux n'est donc pas seulement le signe du « rabâchage » propre à l'auteur. Elle est un autre stigmate de la volonté délibérée de Cohen de sauver, en 1968, l'architecture qui n'avait pu être mise en place en 1938. Et ce vestige doit nécessairement attirer la focale interprétative sur l'importance des effets d'échos entre Mangeclous et Solal qui n'ont jamais pu être publiés par l'auteur tels quels. Cohen tenait absolument à ce que la passion d'Ariane et de Solal soit précédée d'un exposé dogmatique qui dénoncerait la théâtralité factice de l'amour. Cet exposé devait être redoublé par celui qu'en fait Solal au cours de la scène de séduction devant Ariane. La référence à Anna Karénine dans les paroles de Solal $(B S, 408)$ souligne d'ailleurs le fait que cet épisode était à l'origine précédé de l'exposé du Céphalonien et qu'il n'était pas besoin de la développer plus longuement.

À l'heure actuelle, il est donc difficile de ressentir cette structure globale du texte mais il est néanmoins important d'en être conscient. En outre, certains passages des Valeureux ont forcément été écrits après la guerre, n'appartenant donc pas au vaste manuscrit préparatoire des années trente, comme celui où la description de la ruelle d'Or s'interrompt brutalement pour laisser place aux prophéties d'une vieille femme, Belline, qui annonce le destin tragique des Juifs face aux Allemands $(V, 60)$ ou celui où l'auteur prend la parole pour parler du peuple d'Israël et évoquer sa mère morte au cours de la guerre $(V, 91)$. De Mangeclous aux Valeureux, la guerre a donc aussi laissé sa griffe. L'insouciance du premier texte n'était plus permise. Toujours est-il qu'il est désormais nécessaire de lire l'œuvre de Cohen comme un tout, à la manière de celle de Proust, comme un livre unique où les Valeureux ne peuvent être séparés, et encore moins opposés sans nuances, aux amours d'Ariane et de Solal.

\section{Le cabinet aux écritures : essais et tâtonnements de l'intrigue de Belle du Seigneur}

Reste un autre point d'interrogation : le travail de l'écrivain, sur lequel nous n'avons que peu de renseignements puisque tous les avant-textes et les brouillons ont été détruits après son décès. Nous pouvons seulement nous appuyer sur les souvenirs d'un petit nombre de témoins, au rang desquels figure le témoignage très précieux d'Anne-Marie Boissonnas. Son journal montre bien que Belle du Seigneur était en grande partie déjà écrit avant la guerre, et même avant 1938, date de publication de Mangeclous ${ }^{19}$. Il est d'ailleurs à noter que l'intégralité de l'histoire du roman se situe avant la guerre et que Cohen respecte cette donnée même lorsqu'il travaille son texte après le conflit mondial. Le roman s'appelle Belle du Seigneur dès 1937, même si Cohen avait songé à l'appeler Ariane. Les différentes étapes du manuscrit sont rapportées chronologiquement par Anne-Marie Boissonnas.

Sa secrétaire insiste sur la façon de travailler de l'auteur. Il accumulait des notes, des épisodes indépendants qu'il réutilisait le cas échéant. Le texte progresse par ajouts successifs à partir d'un plan bâti à l'avance et que l'écrivain appelle la « carcasse ». Un

19. Anne-Marie Boissonnas-Tillier, « À propos de la première version de Belle du Seigneur (1935-1938) », art. cit., p. 15-24. 
premier manuscrit va voir le jour dans les années 19351937. C'est le premier état du livre que nous connaissons actuellement. En 1935, Cohen n'a pas encore décidé de centrer son roman sur le couple unique de Solal et d'Ariane. Trois femmes occupent le travail de l'écrivain : Yseult, Ariane (l'épouse de Solal) et Diane (la sœur d'Ariane). L'importance du prénom Diane que l'on trouve dans les autobiographies se confirme ici20. La « marche glorieuse »d'Ariane (BS, 648-654), épisode qui transforme l'héroïne en Vierge Marie portée par le bonheur de l'amour, est alors attribuée à Diane. Yseult se suicide à Marseille avant qu'Ariane ne vienne la remplacer. Dans la version finale, Yseult deviendra Isolde (551-519, 524-534), femme âgée et ancienne amante de Solal, qui finira elle aussi par se suicider.

En 1937, Cohen écrit une scène très travaillée, « le péché de vie », racontant l'agonie d'Ariane, femme de Solal, des suites d'un cancer. Diane, sa sœur, à son chevet, connaît avec Solal les prémices d'un grand amour. Le parallèle avec Elizabeth Brocher, première femme de l'écrivain, mourante, et Yvonne Imer, son amie, qui prend son relais, est transparent ${ }^{21}$, d'autant plus que cette scène, supprimée du roman définitif, sera réutilisée dans Le Livre de ma mère en 195422, mais de façon " anonyme », sans qu'aucun personnage n'y soit nommé. Ariane tient le rôle d'Elizabeth mourante et Diane celui d'Yvonne prise d'amour pour Solal-Albert. C'est après la mort d'Ariane que se situe la marche glorieuse de Diane qui célèbre la victoire de l'amour avec Solal. Anne-Marie Boissonnas mentionne l'existence d'autres personnages féminins épisodiques que sont Isabelle et Jane. Ce prénom évoque évidemment Jane Fillon qui, dans le livre à scandale de Nathalie de Saint-Phalle23, se proclamait la véritable Ariane. On voit ainsi que Cohen travaille au départ sur plusieurs figures féminines partiellement inspirées par la réalité qui, au cours des réécritures successives, se fondent peu à peu en une figure composite s'éloignant de plus en plus de la réalité. Il est donc possible que Jane Fillon ait reconnu en Ariane certains de ses traits, puisque cette dernière ne subsume pas moins de cinq personnages féminins (la première Ariane, Diane, Isabelle, Jane et Yseult) mais sans que l'on puisse parler de modèle. C'est en 1937 que Cohen décide de fondre les diverses figures féminines en une seule, Ariane. La publication de
Mangeclous en 1938 le confirme d'ailleurs, puisque le roman annonce dans sa partie finale les amours du couple.

C'est alors que Cohen scinde en deux son manuscrit devenu trop volumineux. Les deux mille trois cents pages sont séparées en deux livres, le premier va jusqu'au départ d'Ariane et de Solal pour Agay (c'est celui qui contient le futur Mangeclous) et le deuxième d'Agay jusqu'à la mort du couple. Comme on le voit, l'essentiel du texte et de l'intrigue est déjà bien en place avant même la publication de Mangeclous. À partir de ce moment, Cohen laisse momentanément de côté le livre II jusqu'en 1938 afin de se consacrer au travail sur le livre I.

Et, si « le cabinet aux écritures n'est pas l'atelier du peintre : on ne le visite pas, mais on peut y pénétrer par effraction 24 », c'est avec Gérard Valbert qu'il convient d'effectuer cette opération délictueuse. Car il a pu consulter dans le détail les carnets tenus par Anne-Marie Boissonnas. Ainsi, il avait connaissance de l'intrigue des premières versions, parfois bien différente de la version définitive. Nous sommes donc en mesure d'esquisser, certes de façon lacunaire, ce que Henri Mitterand appelle une "génétique scénarique », qui évolue selon «un dialogue de l'écrivain avec lui-même 25 ». Les Valeureux

20. Les autobiographies mentionnent souvent une dénommée Diane, symbole de l'amante occidentale sans que rien dans la biographie de l'auteur ne permette de l'identifier (Le Livre de ma mère $(L M)$, Paris, Gallimard, coll. « Folio », 1974 [1954], p. 109-110 ; Carnets 1978, Paris, Gallimard, coll. « Folio », 1992 [1979], p. 91-93). On retrouve plusieurs associations d'Ariane à la Diane mythologique dans Belle du Seigneur (206, 464-465) soulignant bien la proximité de ces deux figures dans l'esprit de l'auteur, comme elles l'ont été dans la genèse du texte. 21. Voir Bella Cohen, Autour d'Albert Cohen, Paris, Gallimard, 1990. 22. «Péché de vie partout. Si la sœur de l'épouse tuberculeuse est saine et jeune, que Dieu ait pitié du beau-frère et de la belle-sœur qui ensemble soignent la malade sincèrement chérie. Ils sont vivants et sains, et lorsque la tuberculeuse dort, sous la morphine et avec un râle souriant, ils vont se promener ensemble dans le jardin nocturne. Ils sont tristes mais ils savourent la douceur du jardin odorant, la douceur d'être ensemble, et c'est presque un adultère » $(L M, 140-141)$.

23. Nathalie de Saint-Phalle, Jane Fillon ou la belle d' un seigneur, Paris, Robert Laffont, 1988.

24. Claude Duchet, "Notes inachevées sur l'inachèvement », dans Almuth Grésillon, Michaël Werner (textes réunis par), Leçons d'écriture. Ce que disent les manuscrits, Paris, Minard, coll. « Lettres modernes », 1985 , p. 243.

25. Henri Mitterand, « Préface », dans Leçons d'écriture. Ce que disent les manuscrits, op. cit., p. X. 
n'apparaissaient qu'au chapitre VIII de ce premier manuscrit. Tout ce qui précédait était chargé de faire le lien entre Solal et Belle du Seigneur en rapportant la vie de Solal entre les deux romans ${ }^{26}$. Tout ceci disparaîtra. C'est un Solal miséreux qui erre dans les rues de Genève, bien différent du glorieux Solal qui ouvrira Belle $d u$ Seigneur en partant à la conquête d'Ariane. Il est invité à déjeuner par la naine Nina - qui deviendra Rachel dans la version finale $(B S, 559-575)^{27}$. Humiliée par l'Armée du Salut ${ }^{28}$, celle-ci raconte son passé en tant que couturière chez des dames de l'aristocratie. Solal lui aussi rapporte sa déchéance. Le fantastique s'immisce dans le texte. Solal, avec une horde de Juifs, erre de pays en pays. Comme on le voit à travers cette partie supprimée par la suite, le fantastique est toujours connexe à la figure de la naine ainsi qu'à l'errance de Solal, comme il l'est dans les versions définitives publiées. Cet épisode est en effet le premier avatar de ce qui deviendra la cave de Berlin où échoue Solal après une errance folle dans les rues de la capitale allemande. De plus, Anne-Marie Boissonnas précise bien qu'en novembre 1937 cet épisode est déjà élaboré. Or, eu égard à la date de publication de Belle du Seigneur, beaucoup de critiques y ont vu un épisode relatant l'holocauste à travers une sorte d'hallucination prophétique de la part des personnages ${ }^{29}$, ce qui, à la lumière de la genèse, paraît difficilement possible. Il est toutefois envisageable que Cohen y ait apporté des modifications après la guerre, renforçant le côté sombre de la scène en raison d'un pessimisme accru lié à la Shoah. Ainsi, ce passage s'annoncerait bien comme la terrible prémonition d'un homme qui voyait les dangers de la montée du nazisme au cours des années trente.

Selon Gérard Valbert, après le premier chapitre où Solal rencontrait la naine et lui racontait ses tribulations de Juif à travers l'Europe, venait le chapitre II où Solal errait, encore une fois, mais à travers Genève ${ }^{30}$. Les intrigues foisonnaient alors. Après avoir essayé de s'introduire à la SDN, Solal s'adresse à Adrien Deume, armé d'une lettre de recommandation de Mme Sarles (personnage de Solal). Celui-ci le méprise. Le Juif pénètre ensuite chez les Deume de force, les menace, oblige Mme Deume à cirer les chaussures des domestiques et M. Deume à chanter l'Internationale. Il les ligote avant d'aller prendre un bain dans la chambre d'Ariane qui devient sa complice. Le roman d'aventure n'est pas loin. Cohen a su par la suite tempérer son exubérance, affiner l'intrigue. Il est ici important de souligner avec Henri Godard que chaque version de l'intrigue « est l'actualisation d'un des possibles du système, dont l'œuvre n'est qu'une réalisation partielle 31 ». Car, « en matière de thématique ou de schèmes d'invention, ce qui a été une fois imaginé et écrit puis abandonné ou transformé n'est pas moins significatif que ce qui a été retenu ». De la sorte, on mesure mieux de quelle manière le romanesque le plus outré est toujours lié au héros qu'est Solal, comme le montre le premier roman de 1930. Néanmoins, cette tendance à la prolifération de l'intrigue sera par la suite refrénée, ce qui donne plus de force à la stagnation délétère dont l'amour est l'enjeu, exténuant au maximum l'intrigue. C'est d'ailleurs le même processus de cristallisation et d'intensification qui se joue dans la fusion des multiples figures féminines en celle d'Ariane, donnant ainsi plus d'unité à l'œuvre et octroyant de fait une place beaucoup plus centrale à la grande passion amoureuse qui conduira Ariane et Solal à la mort. Si ce n'est certains passages de ce premier état du texte qui seront réutilisés dans Mangeclous pour décrire les Deume, on voit bien que Cohen a déjà renoncé à tout ce rocambolesque. Dans le compte rendu de Gérard Valbert, on constate que la plupart des éléments fondamentaux de Belle du Seigneur sont néanmoins présents dès 1937 : le journal intime d'Ariane, la jeune femme dans son bain, la

26. Gérard Valbert, Albert Cohen, le Seigneur, op. cit., p. 309-310.

27. Nina est le prénom qui sera donné finalement à la naine qui apparaît dans le texte autobiographique, Ô vous frères humains, en 1972. L'hésitation entre les deux prénoms montre bien que les deux naines sont similaires, dans l'autobiographie et dans la fiction, ce qui incline à faire des lectures conjointes de ces textes puisqu'ils relèvent du même imaginaire.

28. Les femmes de 1'Armée du Salut ont manifestement exercé une certaine fascination sur Albert Cohen dans ses premiers écrits. Voir à cet égard « Projections ou Après-minuit à Genève », où apparaît aussi, comme une passante baudelairienne, une salutiste.

29. Voir notamment Judith Kauffmann, Grotesque et marginalité. Variations sur Albert Cohen et l'effet-Mangeclous, Bern, Peter Lang, 1999, p. 162-173.

30. Gérard Valbert, Albert Cohen, Le Seigneur, op. cit., p. 310.

31. Henri Godard, « Du bon usage des leçons antérieures », dans Leçons d'écriture, ce que disent les manuscrits, op. cit., p. 148. 
servante Mariette, les dépenses d'Ariane chez le couturier Volkmaar à la manière d'Emma Bovary ruinée, Agay, la comédie des amants parfaits, l'ennui et les remèdes proposés par Solal : faire souffrir Ariane 32 . Il faudra pourtant presque trente ans à l'écrivain pour parvenir à la composition définitive de l'œuvre.

La genèse de Belle du Seigneur est donc bien une genèse contrariée où les impératifs éditoriaux ont joué un rôle majeur quant au contenu et à la structuration des différents romans de Cohen. L'œuvre unique, rêvée par l'auteur, a été un chantier herculéen qui a mobilisé toutes ses forces pendant plus de trente ans. Avec toutes les réécritures, les pages retravaillées, modifiées, amplifiées, Anne-Marie Boissonnas ne décompte en effet pas moins de dix mille pages qu'elle a dactylographiées ${ }^{33}$. Au-delà des variations de détail concernant l'intrigue, l'étude de cette genèse indique que l'imaginaire romanesque de Cohen s'est formé essentiellement dans les années trente et que c'est bien par rapport à ce point de départ qu'il convient d'interroger autant sa pensée, notamment concernant la judéité, que son esthétique, résolument anachronique en 1968 au moment où triomphe le Nouveau Roman. Proposant une réflexion sociale dans la mouvance proustienne ainsi qu'une exploration des arcanes de la psyché en réinvestissant le monologue joycien, Belle du Seigneur conserve les marques du romanesque hyperbolique qui l'a vu naître, dans une tension singulière entre une stagnation de l'intrigue et un tropisme pour le roman d'aventure attaché à la dimension quasi épique et chevaleresque du personnage de Solal. Dans le même temps, la compréhension des mécanismes complexes de la genèse incite à repenser les quatre romans de Cohen comme un tout, liés entre eux dans un jeu de renvoi et de complémentarité signifiant, empêchant tout clivage entre le comique propre aux Valeureux et le tragique qui émerge de la destinée des amants. De la sorte, les échos et parallèles entre Les Valeureux et Mangeclous disent clairement que la structure d'une intrigue n'est jamais accidentelle mais qu'elle fait sens en elle-même. Assurément, une étude génétique est donc particulièrement à même de mettre au jour les enjeux véritables d'une écriture, rendus souterrains et masqués par des contraintes éditoriales extérieures.

32. Gérard Valbert, Albert Cohen, Le Seigneur, op. cit., p. 310-311.

33. Anne-Marie Boissonnas-Tillier, « À propos de la première version de Belle du Seigneur (1935-1938)», art. cit., p. 22. 
MaXime Decout est agrégé de Lettres modernes, enseignant en Khâgne, et titulaire d'une thèse sur la judéité dans l'œuvre d'Albert Cohen, soutenue à l'université Lyon II. Il travaille sur l'œuvre de Cohen et lui a consacré plusieurs articles, notamment dans Les Temps modernes et Poétique, ainsi que sur Perec, auteur à propos duquel il dirige actuellement un numéro spécial de la revue Europe.

Maxime Decout, maximedecout@yahoo.fr

\section{Résumés \\ La genèse contrariée de Belle du Seigneur : le projet déjoué d'une « Geste des Juifs »}

L'œuvre d'Albert Cohen fait assurément partie de celles qui sont entourées du plus grand mystère. L'auteur a en effet pris soin d'éliminer toutes les traces de son activité créatrice, se méfiant des destinées posthumes offertes aux romans. Il a ainsi voulu entretenir le mythe d'une œuvre météore, quasi sui generis. Néanmoins il semble possible de retracer quelques aspects signifiants de la genèse de ces textes. Pénétrer dans les coulisses de la création cohénienne permet alors de comprendre comment les trois derniers romans procèdent en réalité d'une œuvre unique et que les textes publiés sont la résultante de facteurs éditoriaux indépendants de la volonté de l'écrivain. C'est pourquoi l'étude génétique de l'œuvre de Cohen apparaît comme nécessaire pour reconstituer la cohérence d'un projet global, sans laquelle la signification des romans se trouve amoindrie et les lectures partiellement biaisées.

Albert Cohen's work is definitely among those wrapped in the greatest mystery. Indeed, the author took pains to eliminate all traces of his creative activity, as he was wary of novels' posthumous fate. He wanted to keep up the myth of a meteoric work, practically unique of its kind. Nonetheless it seems possible to trace back a few significant aspects of these texts' genesis. By going backstage of the Cohenian creation we can understand that his last three novels spring in fact from a single work and that the published texts are the result of editorial factors independent of the writer's will. That is why a genetic study of Cohen's work seems necessary to reconstitute the coherency of a global project, without which the novels' meaning is reduced and their reading partially distorted.

Das Werk Albert Cohens gehört mit Sicherheit zu jenen Werken, die von einem großen Geheimnis umwoben sind. Der Autor, den postumen Schicksalen von Romanen misstrauend, hat sich in der Tat bemüht, alle Spuren seiner kreativen Tätigkeit zu verwischen. Er wollte auf diese Weise den Mythos eines meteoritenhaften Werkes, quasi sui generis, aufrecht erhalten. Dennoch scheint es möglich, einigen signifikanten Aspekten der Textgenese nachzuspüren. So erlaubt ein Blick hinter die Kulissen des Cohenschen Schaffens, die drei letzten Romane als eine Einheit zu verstehen, deren Veröffentlichung in Form von Einzeltexten editorialen Faktoren geschuldet ist, die nichts mit dem Willen des Autors zu tun haben. Die textgenetische Erforschung von Cohens Werk stellt mithin eine Notwendigkeit dar, um die Kohärenz eines umfassenden Projektes zu rekonstruieren, ohne die der Sinn der Romane verkürzt und ihre Lesarten teilweise verzerrt erscheinen.
La obra de Albert Cohen forma parte, sin duda, de aquellas que aparecen envueltas en un halo de misterio. En efecto, el autor ha eliminado cuidadosamente todas las huellas de su actividad creadora, desconfiando del destino póstumo reservado a las novelas. Ha tratado así de mantener el mito de una obra meteoro, casi sui generis. Sin embargo, se podrían reconstituir algunos aspectos significativos de la génesis de esos textos. Penetrar en el taller creativo de Cohen permitiría entonces entender hasta que punto sus últimas tres novelas proceden en realidad de una obra única y los textos publicados son una resultante de factores editoriales independientes de la voluntad del escritor. Es por ello que el estudio genético de la obra de Albert Cohen se revela necesario para restituir la coherencia de un proyecto global, sin la cual la significación de las novelas resulta menoscabada y las lecturas parcialmente distorsionadas.

A obra de Albert Cohen é, sem dúvida, daquelas que um espesso mistério rodeia. $\mathrm{O}$ autor teve o cuidado de eliminar todos os vestígios da actividade criadora, desconfiando dos destinos póstumos que os romances têm. Pretendeu assim alimentar o mito de uma obra meteórica, quase sui generis. No entanto parece possível reconstituir alguns aspectos significativos da génese destes textos. Nos bastidores da criação de Cohen, percebe-se que os seus três últimos romances derivam na realidade de uma obra única e que as versões publicadas são a resultante de factores editoriais independentes da vontade do escritor. É por isso que o estudo genético da obra de Cohen aparece como necessário para reconstituir a coerência de um projecto global, sem o que a significação dos romances resulta apoucada e as suas leituras um tanto desfocadas.

L'opera di Albert Cohen è certamente tra quelle circondate dal più grande mistero. L'autore ha avuto cura di eliminare tutte le tracce della sua attività creatrice, diffidando dei destini postumi offerti ai romanzi. Così ha voluto alimentare il mito di un'opera meteora, quasi sui generis. Nondimeno sembra possibile rintracciare alcuni aspetti significativi della genesi di questi testi. Penetrare dietro le quinte della creazione di Cohen permette di comprendere allora che i tre ultimi romanzi derivano in realtà da un'opera unica e che i testi pubblicati sono la risultante di fattori editoriali indipendenti dalla volontà dello scrittore. Per questa ragione lo studio genetico dell'opera di Cohen appare necessario per ricostituire la coerenza di un progetto globale, senza la quale il significato dei romanzi appare sminuito e le interpretazioni parzialmente falsate. 